\title{
Relative entropy and curved spacetimes
}

\author{
Fabio Ciolli, Roberto Longo, Alessio Ranallo, Giuseppe Ruzzi \\ Dipartimento di Matematica, Università di Roma Tor Vergata \\ Via della Ricerca Scientifica, 1, I-00133 Roma, Italy*
}

\begin{abstract}
Given any half-sided modular inclusion of standard subspaces, we show that the entropy function associated with the decreasing one-parameter family of translated standard subspaces is convex for any given (not necessarily smooth) vector in the underlying Hilbert space. In second quantisation, this infers the convexity of the vacuum relative entropy with respect to the translation parameter of the modular tunnel of von Neumann algebras. This result allows us to study the QNEC inequality for coherent states in a free Quantum Field Theory on a stationary curved spacetime, given a KMS state. To this end, we define wedge regions and appropriate (deformed) subregions. Examples are given by the Schwarzschild spacetime and null translated subregions with respect to the time translation Killing flow. More generally, we define wedge and strip regions on a globally hyperbolic spacetime, so to have non trivial modular inclusions of von Neumann algebras, and make our analysis in this context.
\end{abstract}

\footnotetext{
${ }^{*}$ Supported by the ERC Advanced Grant 669240 QUEST "Quantum Algebraic Structures and Models", MIUR FARE R16X5RB55W QUEST-NET and GNAMPA-INdAM.
} 


\section{Introduction}

Entropy/energy inequalities lie at the basis of Physics and are currently a subject of great interest in Quantum Field Theory, with motivations coming from diverse sources as black hole thermodynamics, quantum information, conformal field theory. The Quantum Null Energy Condition, QNEC, states that the second derivative of the relative entropy is non-negative

$$
\frac{d^{2}}{d \lambda^{2}} S(\lambda) \geqslant 0
$$

when one shrinks a wedge region in the null direction ( $\lambda$ is a deformation parameter), see [8]. Here $S$ is the relative entropy of the associated observable von Neumann algebra between the vacuum state and any other normal state, see [1]. This a thought-provoking inequality, untrue for general classical or quantum systems, whose ultimate nature is not yet fully understood. Certainly, quantum aspects and relativistic invariance play a role and Operator Algebras provide the right mathematical framework to describe it.

Now, the classical Null Energy Condition is relevant in General Relativity, so one is naturally led to check the QNEC for Quantum Field Theory on a Curved Spacetime. The purpose of this paper is formulate and provide an analysis of the QNEC in this general context. Indeed we shall give a very general result that can be applied to a variety of situations.

In the second quantisation Minkowski context, a formula for the vacuum relative entropy of a coherent state has been defined in [28, 15] and is expressed in first quantisation. The general formula, recalled here below (11), depends on the symplectic structure of the one-particle space, the metric structure plays a role only about the choice of the unitary evolution associated with the Killing flow. This strongly suggests the analysis in [15] be naturally done within a curved spacetime framework.

Our basic result concerns inclusions of standard subspaces of a complex Hilbert space $\mathcal{H}$. Recall that a standard subspace $H$ of $\mathcal{H}$ is a closed, real linear subspace such that

$$
H \cap i H=\{0\}, \quad \overline{H+i H}=\mathcal{H} .
$$

An inclusion of standard subspaces $K \subset H$ is said to be half-sided modular if

$$
\Delta_{H}^{-i s} K \subset K, \quad s \geqslant 0,
$$

with $\Delta_{H}$ the modular operator of $H$, see [27. Then one has a monotone family of translated standard subspaces $H_{s}, s \in \mathbb{R}$, such that $\Delta_{H}^{-i t} K=H_{e^{2 \pi t}}$.

Now, given a vector $\phi \in \mathcal{H}$, the entropy of $\phi$ with respect to the standard subspace $H$ is defined by

$$
S_{\phi}^{H}=\Im\left(\phi, P_{H} i \log \Delta_{H} \phi\right)
$$

(as quadratic form), with $P_{H}$ the cutting projection $H+H^{\prime} \rightarrow H$ [15], see Sect. 2.1. blueHere $H^{\prime} \equiv i H^{\perp}$ is the symplectic complement of $H$, where $H^{\perp}$ is the orthogonal of $H$ w.r.t. to the real part of the scalar product. $S_{\phi}^{H}$ is finite for a dense linear subspace of $\mathcal{H}$ (for the moment we assume $H$ to be factorial, i.e. $H \cap H^{\prime}=\{0\}$ ). 
If $K \subset H$ is half-sided modular, we may consider the function

$$
S_{\phi}^{\mathcal{H}}: \lambda \mapsto S_{\phi}^{H_{\lambda}}
$$

with $\mathcal{H}$ the triple $(\mathcal{H}, H, K)$. Due to general properties of the entropy, $S_{\phi}^{\mathcal{H}}(\lambda) \geqslant 0$ or $S_{\phi}^{\mathcal{H}}(\lambda)=+\infty$ and $S_{\phi}^{\mathcal{H}}(\lambda)$ is non decreasing.

Our main abstract result is that $S_{\phi}^{\mathcal{H}}(\lambda)$ is a convex function for every $\phi \in \mathcal{H}$. Indeed, if for $\lambda_{0} \in \mathbb{R}$ we have $S_{\phi}^{\mathcal{H}}\left(\lambda_{0}\right)<\infty$, then $S_{\phi}^{\mathcal{H}}(\lambda)$ is finite $C^{1}$ on $\left[\lambda_{0}, \infty\right)$ and, on this interval, $\frac{d}{d \lambda} S_{\phi}^{\mathcal{H}}$ is absolutely continuous with $\frac{d^{2}}{d \lambda^{2}} S_{\phi}^{\mathcal{H}} \geqslant 0$ almost everywhere.

The key point about the entropy $S_{\phi}^{H}$ of a vector $\phi$ with respect to the standard subspace $H$ is that it gives Araki's relative entropy

$$
S_{\phi}^{H}=S^{\mathcal{R}_{\varphi}(H)}\left(\varphi_{\phi} \| \varphi\right)
$$

between the vacuum state $\varphi$ and the coherent state $\varphi_{\phi}$ associated with $\phi \in \mathcal{H}$, on the von Neumann algebra $\mathcal{R}_{\varphi}(H)$, on the Bose Fock space, generated by the Weyl unitaries associated with vectors in $H$.

It is now clear how the result immediately gives the QNEC inequality for coherent vectors in a free Quantum Field Theory in a large class of contexts: it suffices to have a globally hyperbolic spacetime, a subregion mapped into itself by a timelike Killing flow in the positive direction and a KMS state for the observable operator algebra. We shall illustrate this geometric operator algebraic structure in the last section, with various examples. We introduce the notions of wedge and strip regions of a globally hyperbolic spacetime and make an analysis in this general framework.

\section{Entropy and modular inclusions}

In this section we make an abstract analysis that will later be applied in the context of Quantum Field Theory on a curved spacetime.

\subsection{Entropy and standard subspaces}

We recall a few facts about standard subspaces and the notion of entropy of a vector with respect to a for standard subspace. References for this section are [27, 15].

Given a complex Hilbert space $\mathcal{H}$, a closed real linear subspace $H$ is said to be standard if $H \cap i H=\{0\}$ and $\overline{H+i H}=\mathcal{H}$. To a standard subspace $H$ one associates an involutive, closed, anti-linear operator, the Tomita operator $S_{H}$

$$
S_{H}(\phi+i \psi)=\phi-i \psi, \phi, \psi \in H,
$$

whose polar decomposition $S_{H}=J_{H} \Delta_{H}$ gives an antiunitary operator $J_{H}$, the modular conjugation, and a self-adjoint, positive, nonsingular operator $\Delta_{H}$, the modular operator, satisfying the relations

$$
J_{H}=J_{H}^{*}, \quad J_{H} \Delta_{H} J_{H}=\Delta_{H}^{-1} .
$$


The modular unitary group is the one-parameter unitary group $\Delta_{H}^{i s}$ that verifies

$$
\Delta_{H}^{i s} H=H, \quad J_{H} H=H^{\prime},
$$

where $H^{\prime}$ denotes the symplectic complement of $H$ given by

$$
H^{\prime}=\{\psi \in \mathcal{H}: \Im(\psi, \phi)=0, \forall \phi \in H\}
$$

We have:

- If $U$ is a unitary operator of $\mathcal{H}$ then $K=U H$ is a standard space and

$$
\Delta_{K}=U \Delta_{H} U^{*}, J_{K}=U J_{H} U^{*} .
$$

- If $H, K$ are standard subspaces of the Hilbert spaces $\mathcal{H}$ and $\mathcal{K}$, then $H \oplus K$ is a standard space of $\mathcal{H} \oplus \mathcal{K}$ and

$$
J_{H \oplus K}=J_{H} \oplus J_{K} \quad, \quad \Delta_{H \oplus K}=\Delta_{H} \oplus \Delta_{K} .
$$

- $H \cap H^{\prime}$ is equal to $\operatorname{ker}\left(1-\Delta_{H}\right)$.

We say that $H$ is factorial if $H \cap H^{\prime}=\{0\}$. Given a factorial standard subspace $H$ of $\mathcal{H}$, the cutting projection associated with $H$ is defined as

$$
P_{H}\left(\phi+\phi^{\prime}\right)=\phi, \quad \phi \in H, \phi^{\prime} \in H^{\prime} .
$$

It turns out that $P_{H}$ is a densely defined, closed, real linear operator satisfying

$$
P_{H}^{2}=P_{H} \quad, \quad-i P_{H} i=P_{i H} \quad, \quad P_{H} \Delta_{H}^{i t}=\Delta_{H}^{i t} P_{H} .
$$

In general, if $H$ is not factorial, we have a direct sum decomposition

$$
H=H_{a} \oplus H_{f},
$$

where $H_{a}=H \cap H^{\prime}$ and $H_{f}$ is the real orthogonal of $H_{a}$. So $\mathcal{H}=\mathcal{H}_{a} \oplus \mathcal{H}_{f}$ where $H_{a} \subset \mathcal{H}_{a}$ is an abelian standard subspace and $H_{f} \subset \mathcal{H}_{f}$ is a factorial standard subspace of $\mathcal{H}_{f}$ (see also [9]).

If $H$ is Abelian, following [9], we recall that the entropy of $\phi$ with respect to $H$ is

$$
S_{\phi}^{H}=2(\phi,(1-E) \phi),
$$

with $E$ the real orthogonal projection of $\mathcal{H}=H+i H$ onto $H$.

In general, if $H \subset \mathcal{H}$ is any standard subspace, we consider the factorial decomposition $H_{a} \oplus H_{f} \subset \mathcal{H}_{a} \oplus \mathcal{H}_{f}$ (2) and define the entropy of $\phi$ with respect to $H$ as

$$
S_{\phi}^{H} \equiv S_{\phi_{a}}^{H_{a}}+S_{\phi_{f}}^{H_{f}}
$$


where $\phi=\phi_{a} \oplus \phi_{f}$ is the decomposition of $\phi, S_{\phi_{a}}^{H_{a}}$ is the relative entropy of $\phi_{a} \in \mathcal{H}_{a}$ with respect $H_{a}$ and similarly for $S_{\phi_{f}}^{H_{f}}$.

If $H \subset \mathcal{H}$ is any closed, real linear subspace, we set $S_{\phi}^{H}=S_{\phi_{s}}^{H_{s}}$, where $H_{s}$ is the standard component of $H$ in $\mathcal{H}_{s}=(H \cap i H)^{\perp_{\mathbb{R}}} \cap \overline{H+i H}$. We thus may, and will, assume the considered subspaces to be standard and factorial.

Some of the main properties of the entropy of a vector are:

- $S_{\phi}^{H} \geqslant 0$ or $S_{\phi}^{H}=+\infty$ (positivity);

- If $K \subset H$, then $S_{\phi}^{K} \leqslant S_{\phi}^{H}$ (monotonicity);

- If $\phi_{n} \rightarrow \phi$, then $S_{\phi}^{H} \leqslant \liminf _{n} S_{\phi_{n}}^{H}$ (lower semicontinuity);

- If $H_{n} \subset H$ is an increasing sequence with $\overline{\bigcup_{n} H_{n}}=H$, then $S_{\phi}^{H_{n}} \rightarrow S_{\phi}^{H}$ (monotone continuity).

It turns out that 15

$$
S_{\phi}^{H}<+\infty \Longleftrightarrow-\int_{0}^{1} \log \lambda d(\phi, E(\lambda) \phi)<+\infty \Longleftrightarrow \phi \in D\left(\sqrt{\left|\log \Delta_{H}\right| E_{-}}\right) .
$$

Here $E(\lambda)$ are the spectral projections of $\Delta_{H}$ and $E_{-}$is the spectral projection onto the negative part of the spectrum of $\log \Delta_{H}$.

Now, let $H$ be a standard subspace of a Hilbert space. Denote by $\mathcal{R}_{\varphi}(H)$ the von Neumann algebra generated by the Weyl unitaries associated with vectors in $H$ on the second quantisation Bose-Fock space over $\mathcal{H}$, and $\varphi$ is the vacuum state (see Section 2.5).

Proposition 2.1. For any standard subspace $H$ of a Hilbert space $\mathcal{H}$ and $\phi \in \mathcal{H}, S_{\phi}^{H}$ equals the Araki's relative entropy

$$
S_{\phi}^{H}=S^{\mathcal{R}_{\varphi}(H)}\left(\varphi_{\phi} \| \varphi\right)
$$

between the restriction of the vacuum state $\varphi$ and of the coherent state $\varphi_{\phi}$ associated with $\phi \in \mathcal{H}$ to the von Neumann algebra $\mathcal{R}_{\varphi}(H)$.

Proof. This proposition is [15, Thm. 4.5] in the factorial case. In the abelian case the proposition is proved in [9]. It then holds in general by considering the factorial decomposition (2). Indeed,

$$
\varphi_{\phi}=\varphi_{\phi_{a} \oplus \phi_{f}}=\varphi_{\phi_{a}} \otimes \varphi_{\phi_{f}}
$$

entails our statements by the additivity of the relative entropy under tensor product. 


\subsection{Half-sided modular inclusions}

We now discuss the properties of the relative entropy of a fixed vector with respect to the one-parameter family of standard subspaces associated with a given half-sided modular inclusion. In particular, we analyse the convexity property.

Let $\mathcal{H}=\left(\mathcal{H}, H_{0}, H_{1}\right)$ be an half-sided modular inclusion i.e. $\mathcal{H}$ is a complex Hilbert space, $H_{1} \subset H_{0}$ is an inclusion of standard subspaces of $\mathcal{H}$ and we have

$$
\Delta_{H_{0}}^{-i s} H_{1} \subset H_{1}, \quad s \geqslant 0 .
$$

By [2, 27, the operator $\frac{1}{2 \pi}\left(\log \Delta_{H_{1}}-\log \Delta_{H_{0}}\right)$ is essentially self-adjoint on $D\left(\log \Delta_{H_{1}}\right) \cap$ $D\left(\log \Delta_{H_{0}}\right)$ with positive closure that we denote by $X$. The one-parameter unitary group generated by $X$, the translation unitary group $U(s)=\exp ($ is $X)$, satisfies the relations

a) $\Delta_{H_{0}}^{-i s} U(s) \Delta_{H_{0}}^{i s}=U\left(e^{2 \pi t} t\right)$ and $J_{H_{0}} U(t) J_{H_{0}}=U(-t), t \in \mathbb{R}$;

b) $U(t) H_{0} \subset H_{0}$ for $t \geqslant 0$;

c) $U(1) H_{0}=H_{1}$

In particular, last relation gives

$$
\Delta_{H_{1}}^{i s}=U(1) \Delta_{H_{0}}^{i s} U(-1) \quad, \quad J_{H_{1}}=U(1) J_{H_{0}} U(-1)
$$

Let $G_{0}$ be the "ax $+b$ " group, namely the group of diffeomorphisms of $\mathbb{R}$ generated by translations and dilations, and let $G$ be the improper " $a x+b$ " group, namely the group generated by $G_{0}$ and the reflection $x \mapsto-x$ on $\mathbb{R}$. Relation $a$ ) says that $\mathcal{H}$ gives a positive energy, anti-unitary representation of $G$. The converse is true [10, namely every positive energy unitary representation of $G$ arises in this way. So, there exists a one-to-one correspondence

Half sided modular inclusion $\longleftrightarrow$ Positive energy anti-unitary representation of $G$

This correspondence concerns representations, not merely unitary equivalence classes. Representations of $G$ with strictly positive energy correspond to factorial half-sided modular inclusions, trivial representations of $G$ to trivial inclusions, i.e. $H_{0}=H_{1}$. Indeed, the following holds:

Proposition 2.2. Let $\mathcal{H}=\left(\mathcal{H}, H_{0}, H_{1}\right)$ be a half-sided modular inclusion and $H_{0}=$ $H_{k, a} \oplus H_{k, f} \subset \mathcal{H}_{k, a} \oplus \mathcal{H}_{k, f}$ the abelian/factorial decomposition of $H_{k}, k=0,1$. Then $\mathcal{H}_{0, a}=\mathcal{H}_{1, a}, \mathcal{H}_{0, f}=\mathcal{H}_{1, f}$ and

$$
H_{a} \equiv H_{0, a}=H_{1, a} .
$$

$H_{a}$ is the fixed-point subspace for the associated unitary representation of $G$.

Proof. Let $H_{a}$ be the $\Delta_{H_{0}}^{i s}$ fixed-point subspace, $s \in \mathbb{R}$, thus $H_{a} \equiv H_{0, a}$. Then $H_{a}$ is pointwise left fixed by $U$ [20, Prop. B.3], with $U$ the associated, positive energy, representation of $G$. By eq. (6),$H_{a}$ is then also left pointwise fixed by $\Delta_{H_{1}}^{i t}$, so $H_{0, a} \subset$ 
$H_{1, a}$. By repeating the argument exchanging $H_{0, a}$ and $H_{1, a}$, we have $H_{1, a} \subset H_{0, a}$, hence the thesis because $U$ has no non-zero fixed vector on $H_{0, f}$ as this is a factorial standard space.

Lemma 2.3. Let $\mathcal{H}=\left(\mathcal{H}, H_{0}, H_{1}\right)$ and $\mathcal{K}=\left(\mathcal{K}, K_{0}, K_{1}\right)$ be half-sided modular inclusions and $T: \mathcal{H} \rightarrow \mathcal{K}$ be a bounded, complex linear operator. The following are equivalent

(i) $T \Delta_{H_{0}}^{i s}=\Delta_{K_{0}}^{i s} T, T \Delta_{H_{1}}^{i s}=\Delta_{K_{1}}^{i s} T, T J_{H_{0}}=J_{K_{0}} T, \quad t \in \mathbb{R}$.

(ii) $T$ intertwines the anti-unitary representations of $G$ associated with $\mathcal{H}$ and $\mathcal{K}$.

Proof. This follows immediately because the anti-unitary representation of $G$ on $\mathcal{H}$ is generated by the modular unitary groups and modular conjugation of $H_{0}, H_{1}$ and similarly for $\mathcal{K}$.

An intertwiner $T$ between two half-sided modular inclusions $\mathcal{H}$ and $\mathcal{K}$ is a bounded, complex linear operator $T: \mathcal{H} \rightarrow \mathcal{K}$ as in the previous lemma. We denote the set of the intertwiners from $\mathcal{H}$ to $\mathcal{K}$ by $(\mathcal{H}, \mathcal{K})$ and say that: $\mathcal{H}$ and $\mathcal{K}$ are unitary equivalent if there is a unitary operator $U \in(\mathcal{H}, \mathcal{K}) ; \mathcal{H}$ is irreducible if the commutant $(\mathcal{H}, \mathcal{H})$ equals $\mathbb{C}$. Given a family of half sided modular inclusions $\mathcal{H}_{\ell}$, their direct sum is the half-sided modular inclusion defined by

$$
\bigoplus_{\ell} \mathcal{H}_{\ell}=\left(\oplus_{\ell} \mathcal{H}_{\ell}, \oplus_{\ell} H_{0 \ell}, \oplus_{\ell} H_{1 \ell}\right) .
$$

As is well known, $G_{0}$ has only one irreducible unitary representation (up to unitary equivalence) with strictly positive energy, the Schrödinger representation; this follows by von Neumann uniqueness theorem on the canonical commutation relations. Every irreducible unitary representation of $G_{0}$ with strictly positive energy is thus a multiple of the Schrödinger representation. Now, the Schrödinger representation of $G_{0}$ extends to an irreducible representation of $G$ on the same Hilbert space (see [27]), we call it with the same name. The following proposition is essentially proved in [27].

Proposition 2.4. Let $U$ be an anti-unitary representation of $G$ with strictly positive energy. Then $U$ is a multiple of the Schrödinger representation. Namely

$$
U=\bigoplus_{\ell} U_{\ell}
$$

where each $U_{\ell}$ is unitary equivalent to the Schrödinger representation.

Proof. Let $U_{0}$ be the restriction of $U$ to $G_{0}$. Thus $U_{0}$ is unitarily equivalent to $V_{0} \otimes 1$ on $\mathcal{H} \otimes \mathcal{K}$, where with $V_{0}$ the Schrödinger representation of $G_{0}$ on $\mathcal{H}$ and $\mathcal{K}$ a Hilbert space. With $V$ the the Schrödinger representation of $G$ on $\mathcal{H}$ extending $V_{0}$, let $J_{0}$ be the anti-unitary involution on $\mathcal{H}$ corresponding to the reflection $x \rightarrow-x$ by $V$, and $J_{1}$ an arbitrary anti-unitary involution on $\mathcal{H}$. By identifying the Hilbert spaces of $U$ and $V_{0} \otimes 1$, we have to prove that if $J$ is a anti-unitary involution on $\mathcal{H} \otimes \mathcal{K}$ commuting with $V_{0} \otimes 1$, then there exists a unitary $T$ on $\mathcal{H} \otimes \mathcal{K}$ commuting with $V_{0}$ such that $J_{0} \otimes J_{1}=T J T^{*}$. 
Now, $J\left(J_{0} \otimes J_{1}\right)$ commutes with $V_{0}$, so $J=J_{0} \otimes Z J_{1}$ for some unitary $Z$ on $\mathcal{K}$ with $J_{1} Z J_{1}=Z^{*}$. Since any two anti-unitary involutions on $\mathcal{K}$ are unitarily equivalent, the result follows

So, there is a unique, up to unitary equivalence, irreducible, half-sided modular inclusion whose associated positive energy unitary representation of $G$ is the Schrödinger one; we call it the Schrödinger half-sided modular inclusion.

Corollary 2.5. Let $\mathcal{H}$ be a factorial half-sided modular inclusion. Then $\mathcal{H}$ is a multiple of the Schrödinger representation. Namely

$$
\mathcal{H}=\bigoplus_{\ell} \mathcal{H}_{\ell}
$$

where each $\mathcal{H}_{\ell}$ is unitary equivalent to the Schrödinger representation.

Proof. Immediate by the above discussion.

Clearly, if $\mathcal{H}$ is not factorial, then $\mathcal{H}$ is the direct sum of a multiple of the Schrödinger representation and a multiple of the trivial inclusion.

\subsection{Entropy convexity in the modular family parameter}

Lemma 2.6. With $\mathcal{H}, \mathcal{K}, \mathcal{H}_{\ell}$ half-sided modular inclusions of standard subspaces, we have

(i) $S_{U \phi}^{K}=S_{\phi}^{H}$ for any unitary $U: \mathcal{H} \rightarrow \mathcal{K}$ such that $U H=K$ and $\phi \in \mathcal{H}$.

(ii) If $\mathcal{H}=\oplus_{\ell} \mathcal{H}_{\gamma}$ and $H=\oplus_{\ell} H_{\ell}$, then

$$
S_{\phi}^{H}=\sum_{\ell} S_{\phi_{\ell}}^{H_{\ell}}
$$

for any vector $\phi=\oplus_{\ell} \phi_{\ell} \in \mathcal{H}$.

Proof. ( $i$ ) is immediate. (ii) easily follows by monotone continuity.

Now, given a half-sided modular inclusion $\mathcal{H}=\left(\mathcal{H}, H_{0}, H_{1}\right)$, by means of the associated translation one parameter unitary group, we can define the translated standard subspaces:

$$
H_{\lambda}=U(\lambda) H_{0}, \quad \lambda \in \mathbb{R} .
$$

Note that

$$
H_{\lambda_{2}} \subset H_{\lambda_{1}}, \quad \lambda_{2}>\lambda_{1} \in \mathbb{R},
$$

Clearly,

$$
\Delta_{\lambda}^{i t}=U(\lambda) \Delta_{0}^{i t} U(-\lambda), \quad J_{\lambda}=U(\lambda) J_{0} U(-\lambda)
$$


where $\Delta_{\lambda}, J_{\lambda}$ are the modular operator and conjugation of $H_{\lambda}$. We have

$$
U_{\mathcal{H}}(\lambda) P_{H_{0}}=P_{H_{\lambda}} U_{\mathcal{H}}(\lambda),
$$

Our aim is to study the convexity of the function given by the translated subspaces $H_{\lambda}$

$$
S_{\psi}^{\mathcal{H}}(\lambda)=\Im\left(\psi, P_{H_{\lambda}} i \log \Delta_{H_{\lambda}} \psi\right),
$$

for any given vector $\psi \in \mathcal{H}$.

Lemma 2.7. We have

(i) $S_{U \phi}^{\mathcal{K}}(\lambda)=S_{\phi}^{\mathcal{H}}(\lambda)$ for any unitary $U \in(\mathcal{H}, \mathcal{K})$ and $\phi \in \mathcal{H}$.

(ii) If $\mathcal{H}=\oplus_{\ell} \mathcal{H}_{\ell}$, then

$$
S_{\phi}^{\mathcal{H}}(\lambda)=\sum_{\ell} S_{\phi_{\ell}}^{\mathcal{H}_{\ell}}(\lambda)
$$

for any vector $\phi=\oplus_{\ell} \phi_{\ell} \in \mathcal{H}$.

Proof. Immediate by Lemma 2.6 ,

Theorem 2.8. Let $\mathcal{H}$ be a half-sided modular inclusion and $\phi \in \mathcal{H}$ a vector. The function

$$
S_{\phi}^{\mathcal{H}}: \lambda \in \mathbb{R} \rightarrow S_{\phi}^{H_{\lambda}} \in[0, \infty]
$$

is convex in $[0, \infty)$.

Suppose further that $S_{\phi}^{H_{\lambda_{0}}}<\infty$ for some $\lambda_{0} \in \mathbb{R}$. Then

(i) $S_{\phi}^{\mathcal{H}}(\lambda)$ is finite and $C^{1}$ on $\left[\lambda_{0}, \infty\right)$;

(ii) $\frac{d}{d \lambda} S_{\phi}^{\mathcal{H}}(\lambda)$ is absolutely continuous in $\left[\lambda_{0}, \infty\right)$ with almost everywhere non-negative derivative $\frac{d^{2}}{d \lambda^{2}} S_{\phi}^{\mathcal{H}}(\lambda) \geqslant 0$.

Proof. By considering the factorial decomposition of $\mathcal{H}$, since the abelian part is fixed by the translation unitaries by Prop. 2.2, we may assume that $\mathcal{H}$ is factorial.

The convexity statement follows by Lemmas 2.6, 2.7 and 2.10 because the (finite or infinite) sum of convex functions is convex.

The proof of the remaining statements relies on Theorem 2.13, If $S_{\phi}^{\mathcal{H}}\left(\lambda_{0}\right)$ is finite, then $S_{\phi}^{\mathcal{H}}(\lambda)$ is finite too, $\lambda \geqslant \lambda_{0}$, by the monotonicity of the entropy. By replacing $H$ with $H_{\lambda_{0}}$, we may assume that $\lambda_{0}=0$. Now, up to unitary equivalence, $\mathcal{H}=\oplus_{\ell} \mathcal{H}_{\ell}$, with $\mathcal{H}_{\ell}$ the Schrördinger representation, see Section 2.4. So we may assume that $\mathcal{H}_{\ell}$ is actually the Schrördinger representation.

With $\phi=\oplus \phi_{\ell}$ the decomposition of $\phi$, we have $\phi_{\ell}^{\prime 2} \in L^{1}\left(\mathbb{R}_{+}, d x\right)$ and

$$
S_{\phi_{\ell}}^{\mathcal{H}_{\ell}}(\lambda)=\pi \int_{\lambda}^{+\infty}(x-\lambda) \phi_{\ell}^{\prime 2}(x) \mathrm{d} x
$$


Then

$$
S_{\phi}^{\mathcal{H}}(\lambda)=\sum_{\ell} S_{\phi_{\ell}}^{\mathcal{H}_{\ell}}(\lambda)=\sum_{\ell} \pi \int_{\lambda}^{+\infty}(x-\lambda) \phi_{\ell}^{\prime 2}(x) \mathrm{d} x=\pi \int_{\lambda}^{+\infty}(x-\lambda) f(x) \mathrm{d} x,
$$

with $f=\sum_{\ell} \phi_{\ell}^{\prime 2}$ almost everywhere. Thus $f \in L^{1}\left(\mathbb{R}_{+}, d x\right)$, so $S_{\phi}^{\mathcal{H}}$ is differentiable on $[0, \infty)$ and the derivative

$$
\frac{d}{d \lambda} S_{\phi}^{\mathcal{H}}(\lambda)=-\pi \int_{\lambda}^{+\infty} f(x) \mathrm{d} x
$$

is continuos. Indeed, $\frac{d}{d \lambda} S_{\phi}^{\mathcal{H}}$ is absolutely continuous and

$$
\frac{d^{2}}{d \lambda^{2}} S_{\phi}^{\mathcal{H}}(\lambda)=\pi f(\lambda)
$$

almost everywhere as desired.

\subsection{The Schrödinger representation}

In order to analyse the entropy properties associated with any (non smooth) vector and the modular family, we need to study a specific representation. Our results in this section extends those in [29], see also [9] for further results.

We realise the Schrödinger representation in the Hilbert space $\mathcal{H}$ is $L^{2}\left(\mathbb{R}_{+}, p d p\right)$. Given $g \in L^{2}\left(\mathbb{R}_{+}, p d p\right)$, we extend $g$ to a function $\tilde{g} \in L^{2}(\mathbb{R},|p| d p)$ by setting $\tilde{g}(-p)=$ $\overline{g(p)}, p>0$. Clearly $\tilde{g}$ is a tempered distribution whose Fourier anti-transform is a real tempered distribution. Call $\mathfrak{L}$ the set of the so obtained distributions:

$$
\mathfrak{L}=\left\{\phi \in \mathcal{S}_{\text {real }}^{\prime}(\mathbb{R}):\left.\hat{\phi}\right|_{[0, \infty)} \in L^{2}\left(\mathbb{R}_{+}, p d p\right)\right\}
$$

So $\mathfrak{L}$ is a real linear space and the Fourier transform gives a real linear, one-to-one identification of $\mathfrak{L}$ with $\mathcal{H}$ (as real vector spaces)

$$
\left.\phi \in \mathfrak{L} \mapsto \hat{\phi}\right|_{[0, \infty)} \in \mathcal{H}
$$

As subspace of $\mathfrak{L}$, the space $C_{0}^{\infty}(\mathbb{R})$ of real, compactly supported, smooth functions on $\mathbb{R}$ embeds into into $\mathcal{H}$, with dense range.

The translation and dilation one-parameter unitary groups $U$ and $V$ are given as follows and define an irreducible, unitary representation of the group $G$ with positive energy. We have

$$
(U(s) \phi)(x)=\phi(x-s), \quad(V(t) \phi)(x)=e^{-t} \phi\left(e^{t} x\right), \quad \phi \in \mathfrak{L},
$$

so $V(t) U(s)=U\left(e^{-t} s\right) V(t)$. The anti-unitary reflection $J$ is given by $(J \phi)(x)=\phi(-x)$, $\phi \in \mathfrak{L}$.

For every $\lambda \in \mathbb{R}$, we set

$$
H_{\lambda}=\{\phi \in \mathfrak{L}: \operatorname{supp}(\phi) \subset[\lambda, \infty)\} .
$$


Then $H_{\lambda}$ is a standard subspace of $\mathcal{H}$ and $C_{0}^{\infty}([0, \infty))$ embeds as a dense, real linear subspace of $H_{\lambda}$. Clearly $U(\lambda) H_{0}=H_{\lambda}$ and these standard subspaces are associated with the Schrödinger half sided modular inclusion $\mathcal{H}=\left(\mathcal{H}, H_{0}, H_{1}\right)$.

The following proposition is proved in [29] (actually, the formula in [29, Sect. 4] is more general, for sectors). We begin by giving a simpler proof here.

Proposition 2.9. With $\mathcal{H}$ the Schrödinger representation, we have

$$
S_{\phi}^{\mathcal{H}}(\lambda)=\pi \int_{\lambda}^{+\infty}(x-\lambda) \phi^{\prime 2}(x) \mathrm{d} x, \quad \phi \in C_{0}^{\infty}(\mathbb{R}),
$$

where $\phi^{\prime}$ is the derivative of $\phi$. Therefore

$$
\begin{gathered}
\frac{d}{d \lambda} S_{\phi}^{\mathcal{H}}(\lambda)=-\pi \int_{\lambda}^{+\infty} \phi^{\prime 2}(x) \mathrm{d} x \leqslant 0, \quad \phi \in C_{0}^{\infty}(\mathbb{R}), \\
\frac{d^{2}}{d \lambda^{2}} S_{\phi}^{\mathcal{H}}(\lambda)=\pi \phi^{\prime 2}(\lambda) \geqslant 0, \quad \phi \in C_{0}^{\infty}(\mathbb{R}) .
\end{gathered}
$$

Proof. We have

$$
\begin{aligned}
& \Im(\phi, \psi)=\frac{i}{2} \int \phi^{\prime}(x) \psi(x) d x, \quad \phi \in C_{0}^{\infty}(\mathbb{R}), \\
& \left(i \log \Delta_{H_{0}} \phi\right)(x)=2 \pi x \phi^{\prime}(x), \quad \phi \in C_{0}^{\infty}(\mathbb{R}), \\
& P_{H_{0}} \phi=\phi \chi_{[0, \infty)}, \quad \phi \in C_{0}^{\infty}(\mathbb{R}), \phi(0)=0 .
\end{aligned}
$$

Therefore, if $\phi \in C_{0}^{\infty}(\mathbb{R})$, we have

$$
S_{\phi}^{\mathcal{H}}(0)=2 \pi \Im\left(\phi, P_{H_{0}} i \log \Delta_{H_{0}} \phi\right)=\pi \int_{0}^{\infty} x \phi^{\prime 2}(x) \mathrm{d} x
$$

that implies (10) by translation covariance. The rest of the proposition follows at once.

Lemma 2.10. Theorem 2.8 is true if $\mathcal{H}$ is the Schrödinger half-sided modular inclusion.

Proof. With $\phi \in \mathcal{H}$, we shall show that the function $\lambda \in \mathbb{R} \rightarrow S_{\phi}^{\mathcal{H}}(\lambda)$ is convex. We may assume that there exists $\lambda_{0} \in \mathbb{R}$ with $S_{\phi}^{\mathcal{H}}(\lambda)<\infty$ and show the convexity in $\left(\lambda_{0}, \infty\right)$ where $S_{\phi}^{\mathcal{H}}(\lambda)$ is finite by the monotonicity of the entropy. By (10), this true if $\phi \in C_{0}^{\infty}(\mathbb{R})$. Set $\lambda_{0}=0$ for simplicity.

Let $T=\sqrt{\left|\log \Delta_{H_{0}}\right|} E_{-}$with $E_{-}$the negative $\log \Delta_{H_{0}}$-spectral projection. By (5) we have

$$
S_{\phi}^{\mathcal{H}}(0)<\infty \Leftrightarrow \phi \in D(T) .
$$

Choose a sequence $\phi_{n} \in C_{0}^{\infty}(\mathbb{R})$, with $\operatorname{supp}\left(\phi_{n}\right) \subset[0, \infty)$, such that $\phi_{n} \rightarrow \phi$ in the graph norm of $T$. 
Now,

$$
S_{\phi}^{H_{0}} \geqslant S_{\phi}^{H_{\lambda}}=S_{\phi}^{U(\lambda) H_{0}}=S_{U(-\lambda) \phi}^{H_{0}}, \quad \lambda \geqslant 0,
$$

therefore $U(-\lambda)$ maps $H_{0}$-finite entropy vectors into $H_{0}$-finite entropy vectors, thus $U(-\lambda) D(T) \subset D(T), \lambda \geqslant 0$. By Lemma 2.14, also $U(-\lambda) \phi_{n}$ converges to $U(-\lambda) \phi$ in the graph norm of $T$. By [15], we have

$$
S_{\phi_{n}}^{\mathcal{H}}(\lambda) \rightarrow S_{\phi}^{\mathcal{H}}(\lambda), \quad \lambda \geqslant 0 .
$$

By Prop. 2.9, $S_{\phi_{n}}^{\mathcal{H}}(\lambda)$ is convex. As the pointwise limit of convex functions is convex, we conclude that $S_{\phi}^{\mathcal{H}}(\lambda)$ is convex too.

Let $\mathfrak{L}_{0}$ be the subspace of $\mathfrak{L}$ consisting of the tempered distributions $\phi \in \mathfrak{L}$ such that $\left.\phi^{\prime}\right|_{(0, \infty)}$ is a Borel function such that

$$
\int_{0}^{\infty} x \phi^{\prime}(x)^{2} d x<\infty
$$

so $\left.\phi^{\prime}(x)\right|_{(0, \infty)} \in L^{2}\left(\mathbb{R}_{+}, x d x\right)$. We define the real, positive, biinear form $q$ on $\mathcal{H}$ with domain $D(q)=\mathfrak{L}_{0}$

$$
q(\phi, \psi)=\int_{0}^{\infty} x \phi^{\prime}(x) \psi^{\prime}(x) d x, \quad \phi, \psi \in D(q)
$$

We also write $q(\phi)=q(\phi, \phi)$ for the associated quadratic form.

Lemma 2.11. The above form $q$ is closable.

Proof. We have to show that for any sequence of vectors $\phi_{n} \in D(q)$ such that $\lim _{n \rightarrow \infty} \phi_{n}=$ 0 in $\mathcal{H}$ and $\lim _{n, m \rightarrow \infty} q\left(\phi_{n}-\phi_{m}\right)=0$, we have $\lim _{n \rightarrow \infty} q\left(\phi_{n}\right)=0$. Namely, if $\phi_{n} \in \mathfrak{L}_{0}$,

$$
\begin{aligned}
& \left\|\phi_{n}\right\|^{2}=\int_{0}^{\infty} p\left|\hat{\phi}_{n}(p)\right|^{2} d p \rightarrow 0, \\
& \int_{0}^{\infty} x\left(\phi_{n}^{\prime}(x)-\phi_{m}^{\prime}(x)\right)^{2} d x \rightarrow 0 \\
& \Rightarrow \int_{0}^{\infty} x \phi_{n}^{\prime}(x)^{2} d x \rightarrow 0 .
\end{aligned}
$$

Now, (14) means that $\phi_{n}^{\prime}$ is a Cauchy sequence in $L^{2}\left(\mathbb{R}_{+}, x d x\right)$ so there exists $f \in$ $L^{2}\left(\mathbb{R}_{+}, x d x\right)$ such that

$$
\phi_{n}^{\prime} \rightarrow f \quad \text { in } L^{2}\left(\mathbb{R}_{+}, x d x\right) .
$$

Eq. (13) means that $\hat{\phi}_{n}$ converges to zero in $L^{2}\left(\mathbb{R}_{+}, p d p\right)$; in particular we have that $\int_{0}^{\infty} \hat{\phi}_{n}(p) \hat{g}(-p) d p \rightarrow 0$ for all $g$ in the Schwartz space $S(\mathbb{R})$, so

$$
\int_{-\infty}^{\infty} \phi_{n}(x) h^{\prime}(x) d p \rightarrow 0, \quad \operatorname{supp}(h) \subset(0, \infty), \quad h \in S(\mathbb{R}),
$$


(integral in distributional sense), thus

$$
\int_{-\infty}^{\infty} \phi_{n}^{\prime}(x) h(x) d x \rightarrow 0, \quad \operatorname{supp}(h) \subset(0, \infty) .
$$

By (16), we then have

$$
\int_{-\infty}^{\infty} f(x) h(x) d x=0,
$$

so $f=0$. Thus (16) implies (15) and $q$ is closable.

Corollary 2.12. Equation (10) holds for all $\phi \in \mathfrak{L}_{0}$. For every $\phi \in \mathcal{H}$, we have

$$
S_{\phi}^{H_{0}}=\int_{0}^{\infty} x \phi^{\prime}(x)^{2} d x
$$

where the right hand side is set equal to $+\infty$ if $\phi^{\prime}$ does not belong to $L^{2}\left(\mathbb{R}_{+}, x d x\right)$.

Proof. Let $\mathcal{D}$ be the set of vectors $\phi \in \mathcal{H}$ such that $S_{\phi}^{H}<\infty$. With $h, k \in \mathcal{D}$, we set

$$
S(h, k)=\Im\left(h, P_{H} i \log \Delta_{H} k\right),
$$

which is well defined by the real polarisation identity. Then $S(\cdot, \cdot)$ is a bilinear form on $\mathcal{D}$ whose associated quadratic form is lower semicontinuous, hence closable. Moreover $C_{0}^{\infty}(\mathbb{R})$ is a form core for $S(\cdot, \cdot)$; this follows because $C_{0}^{\infty}(\mathbb{R})$ is a core for the operator $\sqrt{\log \Delta_{H}} E_{-}$, see [15, Prop. 2.4] and preceding discussion. Since $q$ and $S$ coincide on $\mathcal{D}$, they must agree on the form closure $\mathcal{D}$ by Lemma 2.11 .

Theorem 2.13. Proposition 2.9 holds true for all $\phi \in \mathcal{H}$.

Proof. By Cor. 2.12, we immediately get

$$
S_{\phi}^{H_{\lambda}}=\int_{\lambda}^{\infty}(x-\lambda) \phi^{\prime}(x)^{2} d x,
$$

hence (11), (12) follow by differentiation and clearly entail the theorem.

The following elementary lemma was needed.

Lemma 2.14. Let $\mathcal{H}$ be a Hilbert space, $T: D(T) \subset \mathcal{H} \rightarrow \mathcal{H}$ a closed linear operator. If $U \in B(\mathcal{H})$ maps $D(T)$ into itself, then $\left.U\right|_{D(T)}$ is bounded operator on $D(T)$ with the graph norm of $T$.

Proof. By the closed graph theorem, it suffices to show that $\left.U\right|_{D(T)}$ is closable with respect to the graph norm, namely that

$$
\xi_{n}, \eta \in D(T),\left\|\xi_{n}\right\|+\left\|T \xi_{n}\right\| \rightarrow 0,\left\|U \xi_{n}-\eta\right\|+\left\|T U \xi_{n}-T \eta\right\| \rightarrow 0 \Rightarrow \eta=0,
$$

that holds true because $U$ is bounded, so $\xi_{n} \rightarrow 0$ implies $U \xi_{n} \rightarrow \eta=0$. 


\subsection{Algebras and states associated with a one-particle structure}

We now recall the Weyl algebra and the one-particle structure associated with a quasifree state.

Let $S$ be a symplectic space, that is $S$ a real linear space and $\sigma$ is a symplectic form on $S$; thus $\sigma$ is a real, bilinear, anti-symmetric form on $S \times S$. Given a real scalar product $\mu$ on $S$ satisfying the inequality

$$
\sigma\left(f_{1}, f_{2}\right)^{2} \leqslant \mu\left(f_{1}, f_{1}\right) \cdot \mu\left(f_{2}, f_{2}\right), \quad f_{1}, f_{2} \in S,
$$

a one-particle structure $\left(\mathcal{H}_{\mu}, \kappa_{\mu}\right)$ on $S\left[24\right.$ is given by a complex Hilbert space $\mathcal{H}_{\mu}$ and a real linear mapping $\kappa_{\mu}: S \rightarrow \mathcal{H}_{\mu}$ satisfying

1. $\kappa_{\mu}(S)+i \kappa_{\mu}(S)$ is dense in $\mathcal{H}_{\mu}$,

2. $\Re\left(\kappa_{\mu}\left(f_{1}\right), \kappa\left(f_{2}\right)\right)=\mu\left(f_{1}, f_{2}\right)$ and $\Im\left(\kappa\left(f_{1}\right), \kappa\left(f_{2}\right)\right)=\sigma\left(f_{1}, f_{2}\right)$.

A one-particle structure is unique, modulo unitary equivalence [23].

Now, given a Hilbert space $\mathcal{H}$, we consider the Bose-Fock space $\Gamma(\mathcal{H}):=\bigoplus_{k=0}^{\infty} \mathcal{H}^{\otimes_{s}^{k}}$, where $\mathcal{H}_{0} \equiv \mathbb{C} \xi$ is the one-dimensional Hilbert space of a unit vector $\xi$, the vacuum vector, and $\mathcal{H}^{\otimes_{s}^{n}}$ is the symmetric $n$-fold tensor product of $\mathcal{H}$. To any $\phi \in \mathcal{H}$ there corresponds a coherent vector $e^{\phi}:=\bigoplus_{n=0}^{\infty} \frac{1}{\sqrt{n !}} \phi^{\otimes_{s}^{n}}$ on $\Gamma(\mathcal{H})$, where the zeroth component of $e^{\phi}$ is $\xi$. Coherent vectors form a total family of linearly independent vectors of $\Gamma(\mathcal{H})$, whose scalar product verifies $\left(e^{\phi}, e^{\psi}\right)=e^{(\phi, \psi)}$, for every $\phi, \psi \in \mathcal{H}$.

The Weyl unitaries on $\Gamma(\mathcal{H})$ are first defined on coherent vectors by

$$
W(\psi) e^{\phi}:=e^{\psi+\phi} \cdot e^{-\frac{1}{2}(\psi, \psi)-(\psi, \phi)}, \quad \psi, \phi \in \mathcal{H},
$$

and then extended by linearity and density to all the Fock space. They satisfy the Weyl commutation relations

$$
W(\psi) W(\phi)=e^{-i \Im(\psi, \phi)} W(\psi+\phi), \quad \psi, \phi \in \mathcal{H} .
$$

The Weyl algebra $\mathcal{A}(S)$ associated with the symplectic space $(S, \sigma)$ can be defined as the abstract $\mathrm{C}^{*}$ - algebra generated the Weyl relations, but we shall directly deal with its representation associated with a one-particle structure. Given a one-particle structure $\left(\mathcal{H}_{\mu}, \kappa_{\mu}\right)$ as above, let $\mathcal{A}(S)$ be the $\mathrm{C}^{*}$-algebra obtained as the norm closure of the $*$ algebra generated by the Weyl operators $W_{\mu}\left(\kappa_{\mu}(f)\right)$ as $f$ varies in $S$, where $W_{\mu}$ denotes the Weyl unitaries on $\Gamma\left(\mathcal{H}_{\mu}\right)$.

The (quasi-free) state $\varphi_{\mu}$ of $\mathcal{A}(S)$ associated with $\mu$ is determined by

$$
\omega_{\mu}\left(W_{\mu}\left(\kappa_{\mu}(f)\right)\right)=\left(\xi_{\mu}, W_{\mu}\left(\kappa_{\mu}(f)\right) \xi_{\mu}\right)=e^{-\frac{1}{2}\left\|\kappa_{\mu}(f)\right\|^{2}}=e^{-\frac{1}{2} \mu(f, f)}, \quad f \in S,
$$

with $\xi_{\mu}$ the vacuum vector of $\Gamma\left(\mathcal{H}_{\mu}\right)$. Clearly, it is a normal state of the von Neumann algebra generated by $\mathcal{A}(S)$. 
Note that any real linear, invertible map $T: S \rightarrow S$, that preserves $\sigma$ and $\mu$, promotes to a unitary on $\mathcal{H}_{\mu}$, hence to a unitary $u_{\mu, T}$ on the Fock space $\Gamma\left(\mathcal{H}_{\mu}\right)$ which satisfies

$$
u_{\mu, T} W_{\mu}\left(\kappa_{\mu}(f)\right) u_{\mu, T}^{*}=W_{\mu}\left(\kappa_{\mu}(T f)\right) ;
$$

so $u_{\mu, T}$ implements a vacuum preserving automorphism of $\mathcal{A}(S)$.

\section{Relative entropy and globally hyperbolic spacetimes}

We now make our analysis in the context of globally hyperbolic spacetimes admitting a Killing flow that is timelike and complete within certain causally convex subregions. We first recall some basic facts and then introduce admissible regions so to have, at the second quantisation level, half-sided modular inclusions of von Neumann algebras. We then show that, in the real scalar Klein-Gordon field case, the one-parameter family of relative entropy associated with a coherent state is convex.

We start with a brief description of the causal structure, we refer to [17] for a detailed account [18, 39, 32] and for standard textbooks. Let $\mathcal{M}$ be a 4-dimensional spacetime i.e. a connected, time-oriented, Lorentzian manifold. For any subset $A \subset \mathcal{M}$, the symbols $I^{+}(A), J^{+}(A)$ and $D^{+}(A)$ denote the chronological future, the causal future and the future domain of dependence of $A$. The corresponding past notions will be denoted by replacing + with - .

A subset $A$ of $\mathcal{M}$ is achronal $I^{+}(A) \cap A=\varnothing$. The edge of an achronal set $A$ is the set of the points $p$ in the closure $\bar{A}$ such that for any neighbourhood $U$ of $p$ there are $p_{ \pm} \in I^{ \pm}(p, U)$ joined by a timelike curve contained in $U$ not intersecting $A$; here $I^{ \pm}(p, U)$ is the set of points joined by a future/past-directed (f-d/p-d) timelike curve starting from $p$ and contained in $U$. An achronal subset $S$ such that $D^{+}(S) \cup D^{-}(S)=\mathcal{M}$ is called a Cauchy surface of $\mathcal{M}$. A spacetime $\mathcal{M}$ is globally hyperbolic if one of the following equivalent properties is verified:

(i) $\mathcal{M}$ admits a Cauchy surface;

(ii) $\mathcal{M}$ admits a spacelike Cauchy surface;

(iii) the collection $\left\{I^{+}(p) \cap I^{-}(q), p, q \in \mathcal{M}\right\}$ is a base of neighbourhood for the topology of $\mathcal{M}$ and the sets $J^{+}(p) \cap J^{-}(q)$ are compact for any pair of points $p, q \in \mathcal{M}$. A subset $A \subset \mathcal{M}$ is causally convex whenever $J^{+}(A) \cap J^{-}(A)=A$. The causal convex hull $\operatorname{ch}(S)$ of the set $S$ is the smallest causally convex set containing $S$. Clearly

$$
\operatorname{ch}(S)=J^{+}(S) \cap J^{-}(S), \quad S \subset \mathcal{M} .
$$

By property (iii), any open causally convex subset of a globally hyperbolic spacetime $\mathcal{M}$ is itself a globally hyperbolic spacetime.

\subsection{Wedges and strips}

Generalisation of wedge shaped regions of the Minkowski spacetime to curved spacetimes have been studied by several authors, see [16] and references therein. Here, we give 
notions more suited to our aims.

Within this section we consider a connected globally hyperbolic spacetime $\mathcal{M}$ endowed with a Killing flow $\Lambda$, i.e. a one-parameter group of isometries.

Definition 3.1. A wedge of $\mathcal{M}$ associated with the Killing flow $\Lambda$ is an open connected, causally convex subset $\mathcal{W}$ of $\mathcal{M}$ such that:

(i) $\Lambda_{s}: \mathcal{W} \rightarrow \mathcal{W}$ is a diffeomorphism, $s \in \mathbb{R}$,

(ii) $\Lambda$ is timelike, time oriented and complete within $\mathcal{W}$,

where time oriented means that the Killing vector field generating $\Lambda$ is either f-d for all points of $\mathcal{W}$ or $p$-d for all points of $\mathcal{W}$.

Note that, since a wedge $\mathcal{W}$ is open and causally convex, $\mathcal{W}$ is itself a globally hyperbolic spacetime with the induced Lorentzian structure; as $\Lambda$ is assumed to be timelike complete, $\mathcal{W}$ is indeed a globally hyperbolic stationary spacetime.

We set

$$
\mathrm{O}_{A}:=\left\{\Lambda_{s}(p): p \in A, s \in \mathbb{R}\right\},
$$

for the orbit of the set $A \subset \mathcal{W}$. We also consider the positive/negative half orbit $\mathrm{O}_{A}^{ \pm}:=$ $\left\{\Lambda_{s}(p): p \in A, \pm s>0\right\}$.

Lemma 3.2. Let $\mathcal{W}$ be a wedge of $\mathcal{M}$ associated with $\Lambda$. For any $p \in \mathcal{W}$,

$$
\mathcal{W}=\operatorname{ch}\left(\mathrm{O}_{p}\right)=I^{-}\left(\mathrm{O}_{p}\right) \cap I^{+}\left(\mathrm{O}_{p}\right) .
$$

Proof. Without loss of generality we assume that $\Lambda$ is f-d. We prove that $I^{-}\left(\mathrm{O}_{p}\right) \cap$ $I^{+}\left(\mathrm{O}_{p}\right)=\operatorname{ch}\left(\mathrm{O}_{p}\right)$. To this end note that $I^{+}\left(\mathrm{O}_{p}\right) \cap I^{-}\left(\mathrm{O}_{p}\right)$ is causally convex (this easily follows by the properties of causal sets). Hence $\operatorname{ch}\left(\mathrm{O}_{p}\right) \subset I^{+}\left(\mathrm{O}_{p}\right) \cap I^{-}\left(\mathrm{O}_{p}\right)$. Conversely, given $x \in I^{+}\left(\mathrm{O}_{p}\right) \cap I^{-}\left(\mathrm{O}_{p}\right)$ there are $s_{1}, s_{2} \in \mathbb{R}$ such that $s_{1}<s_{2} x \in$ $I^{+}\left(\Lambda_{s_{1}}(p)\right) \cap I^{-}\left(\Lambda_{s_{2}}(p)\right)$. Taking $s_{3}<s_{1}<s_{2}<s_{4}$, we have that

$$
x \in I^{+}\left(\Lambda_{s_{1}}(p)\right) \cap I^{-}\left(\Lambda_{s_{2}}(p)\right) \subset J^{+}\left(\Lambda_{s_{3}}(p)\right) \cap J^{-}\left(\Lambda_{s_{4}}(p)\right) \subset \operatorname{ch}\left(\mathrm{O}_{p}\right) .
$$

So $I^{-}\left(\mathrm{O}_{p}\right) \cap I^{+}\left(\mathrm{O}_{p}\right)=\operatorname{ch}\left(\mathrm{O}_{p}\right)$ and $I^{-}\left(\mathrm{O}_{p}\right) \cap I^{+}\left(\mathrm{O}_{p}\right) \subset \mathcal{W}$ because $\mathcal{W}$ is causally convex. On the other hand, as $\mathcal{W}$ is a globally hyperbolic stationary spacetime with respect to $\Lambda$, we have

$$
\mathcal{W}=I^{-}\left(\mathrm{O}_{p}, \mathcal{W}\right)=I^{+}\left(\mathrm{O}_{p}, \mathcal{W}\right)
$$

In fact, taking a smooth spacelike Cauchy surface $\mathcal{C}$ containing $p$, the mapping $\mathbb{R} \times \mathcal{C} \ni$ $(s, x) \mapsto \Lambda_{s}(x) \in \mathcal{W}$ is an isometry if $\mathbb{R} \times \mathcal{C}$ is equipped with the induced metric

$$
g_{(t, x)}=-\beta(x) d t^{2}+2 \omega_{x} d t+h_{x}, \quad(t, x) \in \mathbb{R} \times \mathcal{C},
$$

where $\omega$ is a 1 -form, $\beta$ is a smooth strictly positive function, $h$ is a Riemannian metric over $\mathcal{C}[13$, Theorem 2.3]. Then, for any point $q$ in $\mathcal{C}$ take a spacelike curve $\alpha:[0,1] \rightarrow \mathcal{C}$ with $\alpha(0)=q$ and $\alpha(1)=p$. As $\beta$ is positive and depends only on $\mathcal{C}$, and as $\Lambda$ is complete, by continuity and compactness of $[0,1]$ one can always find a smooth increasing function 
$f:[0,1] \rightarrow(0,+\infty)$ such that the curve $\rho(t):=(f(t), \alpha(t))$ is $\mathrm{f}$-d timelike. Clearly $\rho(0)=q$ and $\rho(1)=\Lambda_{f(1)}(p)=(f(1), p)$. So $q \in I^{-}\left(\mathrm{O}_{p}, \mathcal{W}\right)$ and as this holds for any point of $\mathcal{C}$ we have that $I^{-}\left(\mathrm{O}_{p}, \mathcal{W}\right)=\mathcal{W}$. Therefore,

$$
\mathcal{W}=I^{-}\left(\mathrm{O}_{p}, \mathcal{W}\right) \cap I^{+}\left(\mathrm{O}_{p}, \mathcal{W}\right) \subset I^{-}\left(\mathrm{O}_{p}\right) \cap I^{+}\left(\mathrm{O}_{p}\right) \subset \mathcal{W}
$$

completing the proof.

Given a wedge $\mathcal{W}$ of $\mathcal{M}$ associated with $\Lambda$, we say that an open connected subset $\mathcal{V} \subset \mathcal{W}$ is (positively) half-invariant w.r.t. $\Lambda$ whenever

$$
\Lambda_{s}(\mathcal{V}) \subset \mathcal{V}, \quad \forall s>0 .
$$

Note that, given a $\Lambda$ half-invariant subset $\mathcal{V}$, then $\Lambda_{s}(\mathcal{V})$ is half-invariant too, $s>0$.

We are going to consider two kind of half-invariant subregions of a wedge $\mathcal{W}$ of $\mathcal{M}$. Let $\tau$ be an isometry of $\mathcal{M}$. The image $\tau(\mathcal{W}) \equiv \mathcal{W}^{\tau}$ is a wedge with the respect to

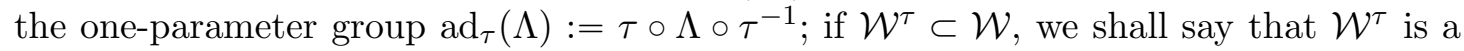
subwedge of $\mathcal{W}$.

Now, $\mathcal{W}^{\tau}$ is $\Lambda$ half-invariant whenever $\Lambda_{s}(\tau(\mathcal{W})) \subset \tau(\mathcal{W})$ for $s>0$. By Lemma (3.2)

$$
\mathcal{W}^{\tau}=\operatorname{ch}\left(\left\{\operatorname{ad}_{\tau}\left(\Lambda_{s}\right)(\tau(p)), s \in \mathbb{R}\right\}\right)=\operatorname{ch}\left(\left\{\tau \circ \Lambda_{s}(p), s \in \mathbb{R}\right\}\right)=\tau\left(\operatorname{ch}\left(\mathrm{O}_{p}\right)\right)
$$

for any $p \in \mathcal{W}$, so we have

$$
\left(\Lambda_{t} \circ \tau\right)\left(\mathrm{O}_{p}\right) \subset \tau\left(\operatorname{ch}\left(\mathrm{O}_{p}\right)\right) \Longleftrightarrow \operatorname{ad}_{\tau^{-1}} \circ \Lambda_{t}\left(\mathrm{O}_{p}\right) \subset \operatorname{ch}\left(\mathrm{O}_{p}\right) .
$$

Even though the spacetime has no symmetries besides $\Lambda$, there are always proper halfinvariant subregions of $\mathcal{W}$ that, in general, are neither wedges nor causally convex. We now give an interesting family of such regions.

Definition 3.3. Given a connected achronal subset $A$ of $\mathcal{W}$ with $A \cap \operatorname{edge}(A)=\varnothing$, the half-orbit $\mathrm{O}_{A}^{+}$is called the (positive) strip generated by $A$.

Lemma 3.4. Let $A \subset \mathcal{W}$ be a connected achronal such that $A \cap \operatorname{edge}(A)=\varnothing$. Then $\mathrm{O}^{+}(A)$ is an open connected half-invariant subset of $\mathcal{W}$.

Proof. We assume that $\Lambda$ is $\mathrm{f}-\mathrm{d}$ and $\mathrm{O}_{A}^{+}$is clearly connected. Furthermore as $A$ is achronal and $\Lambda$ timelike then $A \cap \Lambda_{s}(A)=\varnothing$ for any $s>0$, so

$$
\Lambda_{s_{1}}(A) \cap \Lambda_{s_{2}}(A)=\varnothing, \quad 0<s_{1}<s_{2} .
$$

So $\mathrm{O}_{A}^{+}$is half-invariant w.r.t. $\Lambda$. What remains to be shown is that $\mathrm{O}_{A}^{+}$is open. Given $p \in \mathrm{O}_{A}^{+}$, then $p \in \Lambda_{s_{*}}(A) \equiv A_{s_{*}}$ for some $s_{*}>0 . A_{s_{*}}$ is achronal and $A_{s_{*}} \cap \operatorname{edge}\left(A_{s_{*}}\right)=$ $\varnothing$. The latter, in particular, implies that we can find a relatively compact subset $U$ s.t. $p \in U \subset \bar{U} \subset A_{s_{*}}$ and

$$
U \cap \operatorname{edge}(U)=\varnothing
$$


By [4] there is an achronal Cauchy surface $\mathcal{C}$ containing the closure $\bar{U}$ of $U$. Then as $\Lambda$ is timelike and complete, the mapping

$$
\mathbb{R} \times \mathcal{C} \ni(s, x) \mapsto \Lambda_{s}(x) \in \mathcal{M}
$$

is an homeomorphism [3, Lemma 2]: it is continuous and bijective because for any point $p \in \mathcal{M}$ the timelike curve $\Lambda_{t}(p)$ meets $\mathcal{C}$ exaclty once. The invariance of domain theorem implies that this mapping is a homeomorphism.

As $U \cap \operatorname{edge}(U)=\varnothing$, we have that $U$ is open in $\mathcal{C}$. It is enough to observe that the limit points of any sequence $x_{n} \in \mathcal{C} \backslash U$ belong to $\mathcal{C} \backslash U$ because otherwise [30, Proposition 2.140] they would be edge points of $U$ (the sequence $x_{n}$ is in the complement of $I^{+}(U) \cup$ $\left.I^{-}(U) \cup U\right)$.

In conclusion, for $\varepsilon>0$ and small enough, the set $\left\{\Lambda_{s}(U): s \in\left(s_{*}-\varepsilon, s_{*}+\varepsilon\right)\right\}$ is an open subset of $\mathrm{O}_{A}^{+}$containing $p$, and this proves that $\mathrm{O}_{A}^{+}$is open.

Note that the causal complement of a strip is empty in the wedge as follows by applying the same reasoning of the proof the Lemma 3.2

\subsubsection{Examples of half invariant regions}

Minkowski spacetime. Let $\mathcal{M}=\mathbb{R}^{4}$ be the 4-dimensional Minkowski spacetime with Poincaré symmetry group. With $\Lambda$ the Killing flow given the pure Lorentz transformations in the $x_{1}$ direction, a wedge with respect to $\Lambda$ is the usual right wedge $\mathcal{W}_{0}=\left\{x \in \mathbb{R}^{4}: x_{1}>\left|x_{0}\right|\right\}$. Let $\mathcal{W}_{0}^{\tau}$ be the translated of $\mathcal{W}_{0}$ by a Poincaré element $\tau$; if $\mathcal{W}_{0}^{\tau}$ is contained in $\mathcal{W}$, then $\mathcal{W}_{0}^{\tau}$ is half-invariant if $\tau$ is a lightlike translation.

Strips are easily defined. We point out that deformed wedges considered in [8], see also [29, 31], can be seen as strips of a suitable achronal subset. For any non-negative smooth function $f(y)$ with $y=\left(x_{2}, x_{3}\right)$, we consider the deformation of the right-wedge $\mathcal{W}_{0}$ defined as

$$
\mathcal{W}_{f}:=\left\{\left(x_{0}, x_{1}, y\right) \in \mathcal{M},\left|x_{0}-f(y)\right|<x_{1}-f(y)\right\} .
$$

This is not a wedge since it is neither causally convex nor $\Lambda$ invariant. However, for any $\lambda>0$, the translated $\mathcal{W}_{f+\lambda}=(\lambda, \lambda, 0,0)+\mathcal{W}_{f}$ is a positive $\Lambda$-invariant subregion of $\mathcal{W}_{f}$. Then, setting

$$
A_{f+\lambda}:=\left\{\left(x_{0}, x_{1}, y\right) \in \mathcal{M} \mid x_{0}=-x_{1}+2(f(y)+\lambda), x_{1}>f(y)+\lambda, y \in \mathbb{R}^{2}\right\},
$$

we get a connected, smooth surface which is an achronal subset of $\mathcal{W}_{0}$ with $A_{f+\lambda} \cap$ edge $\left(A_{f+\lambda}\right)=\varnothing$ in $\mathcal{W}_{0}$. We have $\mathcal{W}_{f+\lambda}=\mathrm{O}_{A_{f+\lambda}}^{+}$, the strip generated by $A_{f+\lambda}$. Therefore, $\mathcal{W}_{f}$ is the translated of a strip: $\mathcal{W}_{f}=\mathrm{O}_{A_{f+\lambda}}^{+}-(\lambda, \lambda, 0,0)$.

Rindler spacetime. The Rindler spacetime is a wedge itself: it may be considered as the above standard wedge of the Minkowski spacetime with hyperbolic coordinates [15]. 
The Lorentz boosts of Minkowski correspond to time translation in Rindler spacetime giving a time-like Killing flow $\Lambda$ that becomes light-like on the boundary of the wedge (horizons). Strips are defined as in the Minkowski case.

Kruskal spacetime. The Kruskal spacetime $\mathcal{M}$ is a 4-dimensional globally hyperbolic spacetime arising as the maximal analytic extension of the Schwarzschild spacetime. We stare here few basic properties, for further details see e.g. the references at the beginning of this section.

In the Kruskal-Szekeres coordinates the metric is defined on $\left\{(t, x) \in \mathbb{R}^{2}: x^{2}-t^{2}<\right.$ $-1\} \times \mathbb{S}^{2}$ as

$$
d s^{2}=\frac{32 M^{3}}{r} e^{-\frac{r}{2 M}}\left(-d t^{2}+d x^{2}\right)+r^{2} d \Omega^{2}
$$

where $d \Omega^{2}$ the area element of the unit 2 -sphere $\mathbb{S}^{2}, M>0$ is the black hole mass, and $r \in(0,+\infty)$ is the Schwarzschild radius implicitly related to $t, x$ by

$$
x^{2}-t^{2}=e^{\frac{r}{2 M}}\left(\frac{r}{2 M}-1\right) .
$$

The metric has a "physical" singularity at $x^{2}-t^{2}=-1 \Longleftrightarrow r=0$.

There are four Killing vector fields generating the symmetry group: the ones associated with the spatial rotation group $S O(3)$, and the time translation Killing flow that acts on the $(t, x)$ component by

$$
\Lambda_{s}=\left(\begin{array}{cc}
\cosh (s / 4 M) & \sinh (s / 4 M) \\
\sinh (s / 4 M) & \cosh (s / 4 M)
\end{array}\right)
$$

and trivially on the $\mathbb{S}^{2}$ component. $\Lambda$ is timelike in the right wedge $\mathcal{W}_{+}:=\left\{t^{2}-x^{2}>\right.$ $0, x>0\} \times \mathbb{S}^{2}$, corresponding to the external region of the Schwarzschild black hole, and in the left wedge $\mathcal{W}_{-}:=\left\{t^{2}-x^{2}>0, x<0\right\} \times \mathbb{S}^{2}$

We consider the family of strips given by $\mathcal{W}_{+, \lambda}=\mathcal{W}_{+}+(\lambda, \lambda)$, with $\lambda>0$ (translation on the $\mathbb{R}^{2}$ component). $\mathcal{W}_{+, \lambda}$ is indeed a strip since it is the $\Lambda$ half-orbit of the translated right-past horizon $h_{\lambda}:=\left\{x^{2}-t^{2}=0, x>0, t<0\right\} \times \mathbb{S}^{2}+(\lambda, \lambda)$. Moreover, as in the Minkowski case, one can define a deformation $\mathcal{W}_{+, f}$ of $\mathcal{W}_{+}$, for any non negative smooth function $f$ on $\mathbb{S}^{2}$ :

$$
\mathcal{W}_{+, f}=\{(t, x, \Omega) \in \mathcal{M},|t-f(\Omega)|<x-f(\Omega)\}
$$

whose $\lambda$-translated is the strip generated by the achronal set $h_{f+\lambda}$ defined as

$$
h_{f+\lambda}:=\{(t, x, \Omega) \in \mathcal{M} \mid t=-x+2(f(\Omega)+\lambda), x>f(\Omega)+\lambda\} .
$$

Further examples. As is known, wedge regions of the $d$-dimensional de Sitter spacetime $d S^{d}$ are naturally defined by the embedding of $d S^{d}$ into in $\mathbb{R}^{1+d}$; strips can be similarly defined. Concerning the Minkowski spacetime $\mathcal{M}$ with time translation Killing flow $T$, then the forward light cone is a $T$-half invariant subregion, indeed a strip of the 
wedge $\mathcal{M}$; in the one-dimensional case, a positive half-line is a half invariant subregion of $\mathbb{R}$. KMS states in these contexts are studied respectively in [33, 7] and [11, 12, 26].

We finally point out that the relative entropy concept in this paper is also applicable to spacetimes describing non-stationary black holes, as recently discussed in [25].

\subsection{Entropy and Klein-Gordon field on a globally hyperbolic space- time}

We now consider Weyl quantisation of the Klein-Gordon free scalar field on a globally hyperbolic spacetime $\mathcal{M}$.

The Klein-Gordon operator is $-\square+m^{2}$, where $\square$ is the D'Alembertian associated with the spacetime metric tensor and $m$ is the mass. We consider the space of compactly supported, smooth functions $C_{0}^{\infty}(\mathcal{M}, \mathbb{R})$ equipped with the symplectic form

$$
\sigma\left(f_{1}, f_{2}\right):=\frac{1}{2} \int_{\mathcal{M}} f_{1} E\left(f_{2}\right) d \nu, \quad f_{1}, f_{2} \in C_{0}^{\infty}(M, \mathbb{R})
$$

where $E$ is the advanced-minus-retarded propagator of $-\square+m^{2}$ and $\nu$ is the metricinduced volume measure on $\mathcal{M}$.

The symplectic form $\sigma$ annihilates on the image of the Klein-Gordon operator. So we consider the quotient $S(\mathcal{M}):=C_{0}^{\infty}(\mathcal{M}, \mathbb{R}) /\left[\left(-\square+m^{2}\right)\left(C_{0}^{\infty}(\mathcal{M}, \mathbb{R})\right)\right]$ where $\sigma$ is non degenerate. For simplicity, in the following we omit the equivalence class symbol when considering elements of $S(\mathcal{M})$.

The Weyl algebra of the Klein-Gordon field is the Weyl $\mathrm{C}^{*}$-algebra associated with the symplectic space $(S(\mathcal{M}), \sigma)$ as in Section 2.5.

We consider a one-particle structure $\left(\mathcal{H}_{\mu}, \kappa_{\mu}\right)$, where $\mu$ is a real scalar product on $S(\mathcal{M})$ as in Section 2.5, For simplicity, we set

$$
W_{\mu}(f):=W_{\mu}\left(\kappa_{\mu}(f)\right), \quad f \in C_{0}^{\infty}(\mathcal{M}) .
$$

and note that the Weyl unitaries satisfy the Klein-Gordon equation

$$
W_{\mu}\left(\left(-\square+m^{2}\right) f\right)=1, \quad f \in C_{0}^{\infty}(\mathcal{M}) .
$$

Given a globally hyperbolic spacetime with a Killing flow $\Lambda$, let $\varphi_{\mu}$ be a quasi-free state, defined by a one-particle structure $\left(\mathcal{H}_{\mu}, \kappa_{\mu}\right)$ which is left invariant by $\Lambda$. For any region $\mathcal{O}$ with nonempty interior of $\mathcal{M}$, we consider the real subspace

$$
H_{\mu}(\mathcal{O}):=\left\{\kappa_{\mu}(f), f \text { compactly supported in } \mathcal{O}\right\}^{-} \subset \mathcal{H}_{\mu}
$$

and the von Neumann algebra

$$
\mathcal{R}_{\mu}(\mathcal{O}):=\left\{W_{\mu}(f), f \text { compactly supported in } \mathcal{O}\right\}^{\prime \prime} .
$$

The correspondence $\mathcal{O} \mapsto \mathcal{R}_{\mu}(\mathcal{O})$ satisfies 
- $\mathcal{O}_{1} \subset \mathcal{O}_{2} \Rightarrow \mathcal{R}_{\mu}\left(\mathcal{O}_{1}\right) \subset \mathcal{R}_{\mu}\left(\mathcal{O}_{2}\right)$ (isotony);

- $\mathcal{O}_{1} \perp \mathcal{O}_{2} \Rightarrow\left[\mathcal{R}_{\mu}\left(\mathcal{O}_{1}\right), \mathcal{R}_{\mu}\left(\mathcal{O}_{2}\right)\right]=0$, (causality);

- $u_{\mu, T} \mathcal{R}_{\mu}(\mathcal{O}) u_{\mu, T}^{*}=\mathcal{R}_{\mu}(\tau \mathcal{O})$ if $\tau$ is an isometry of $\mathcal{M}$ leaving $\mu$ invariant and $T$ is the associated symplectic transformation (see Sect. 2.5) (covariance);

here $\mathcal{O}_{1} \perp \mathcal{O}_{2}$ means that the closure of $\mathcal{O}_{1}$ and $\mathcal{O}_{2}$ are causal disjoint. We note that this representation is irreducible when the real subspace $\kappa_{\mu}(S(\mathcal{M}))$ is dense in $\mathcal{H}_{\mu}$. Other properties of the Weyl algebra of the Klein-Gordon field can be found in [38].

We now assume that $(\mathcal{M}, \Lambda)$ is stationary, i.e. $\Lambda$ is timelike complete. In this case the Weyl algebra of the Klein-Gordon field admits, for any $\beta>0$ and $m>0$, a unique quasi-free $\beta$-KMS Hadamard state [35] with respect to the timelike Killing flow $\Lambda$. In the following, we fix $\beta>0, m>0$; the one-particle structure is the one associated with this quasi-free $\beta$-KMS state and we set $\mu=\beta$.

Proposition 3.5. Let $(\mathcal{M}, \Lambda)$ be a stationary spacetime and let $\varphi_{\beta}$ be a $\beta$-KMS quasifree Hadamard state of the Weyl algebra. Then, if $\mathcal{V}$ is any non-empty half-invariant region of $\mathcal{M}$, the triple $\mathcal{H}_{\boldsymbol{\beta}}:=\left(\mathcal{H}_{\beta}, H_{\beta}(\mathcal{M}), H_{\beta}(\mathcal{V})\right)$ is a half-sided modular inclusion; the relative entropy function

$$
S^{\mathcal{R}_{\beta}\left(\mathcal{V}_{\lambda}\right)}\left(\varphi_{\phi} \| \varphi_{\beta}\right)=S_{\phi}^{\mathcal{H}_{\beta}}(\lambda)
$$

is convex with respect to $\lambda$.

Proof. Let $u_{\beta, s}$ be the unitary on $\Gamma\left(\mathcal{H}_{\beta}\right)$ associated with $\Lambda_{s}$. The $\beta$-KMS condition implies that

$$
\operatorname{ad}\left(u_{\beta, \beta s}\right)=\operatorname{ad}\left(\Delta_{\mathcal{R}_{\beta}(\mathcal{M})}^{-i s}\right),
$$

i.e. the one-parameter automorphism group of the von Neumann algebra $\mathcal{R}_{\beta}(\mathcal{M}):=$ $\mathcal{A}(\mathcal{M})^{\prime \prime}$ on the Fock space is the modular group ad $\Delta_{\mathcal{R}_{\beta}(\mathcal{M})}^{-i s}$.

The von Neumann algebra associated with a non empty, open, relatively compact region satisfies the Reeh-Schlieder property [37]. So, the corresponding real Hilbert space $H_{\beta}(\mathcal{O})$ is a standard subspace of $\mathcal{H}_{\beta}$ and

$$
\Delta_{\mathcal{R}_{\beta}(\mathcal{O})}=\Gamma\left(\Delta_{H_{\beta}(\mathcal{O})}\right), \quad J_{\mathcal{R}_{\beta}(\mathcal{O})}=\Gamma\left(J_{H_{\beta}(\mathcal{O})}\right), \quad \mathcal{O} \subset \mathcal{M},
$$

where $\Delta_{H_{\beta}(\mathcal{O})}$ and $J_{H_{\beta}(\mathcal{O})}$ is the modular operator of $H_{\beta}(\mathcal{O})$. Now, since $\mathcal{V}$ is half invariant, (20) and (21) give

$$
\Delta_{H_{\beta, 0}}^{-i s} H_{\beta}(\mathcal{V})=H_{\beta}\left(\Lambda_{s}(\mathcal{V})\right) \subset H_{\beta}(\mathcal{V}), \quad t>0,
$$

that implies the triple $\mathcal{H}_{\boldsymbol{\beta}}:=\left(\mathcal{H}_{\beta}, H_{\beta}(\mathcal{M}), H_{\beta}(\mathcal{V})\right)$ to be a half-sided modular inclusion. Hence, by Proposition 2.1, we have that

$$
S^{\mathcal{R}_{\beta}\left(\mathcal{V}_{\lambda}\right)}\left(\varphi_{\phi} \| \varphi_{\beta}\right)=S_{\phi}^{\mathcal{H}_{\beta}}(\lambda) .
$$

The convexity then follows by Theorem 2.8 
The above results can be generalized in the context of a globally hyperbolic spacetime $\mathcal{M}$ with a wedge $\mathcal{W}$ with respect to the Killing flow $\Lambda$. Being $(\mathcal{W}, \Lambda)$ globally hyperbolic and stationary, the corresponding Weyl algebra has $\beta$-KMS state $\varphi_{\beta}$. If $\varphi_{\beta}$ admits an extension to a quasi-free $\Lambda$-invariant state $\varphi_{o}$ (the "vacuum state") of the Weyl algebra of $\mathcal{M}$ such that the vacuum vector $\xi_{o}$ is cyclic for the algebra $\mathcal{R}_{o}(\mathcal{W})$, then we may consider the unitary $U_{o, \beta}$ defined by

$$
U_{o, \beta} W_{\beta}(f) \xi_{\beta}:=W_{o}(f) \xi_{o}, \quad f \in C_{0}^{\infty}(\mathcal{W}) ;
$$

then the results of Propositions 3.5 easily extend to the vacuum representation of the Weyl algebra of $\mathcal{M}$.

Corollary 3.6. Let $\mathcal{M}$ be a globally hyperbolic spacetime with a wedge $\mathcal{W}$ with respect a Killing flow $\Lambda$. Let $\varphi_{\beta}$ be a KMS quasi-free state of the Weyl algebra of $\mathcal{W}$ which admits an extension to a quasi-free state $\varphi_{o}$ of the Weyl algebra of $\mathcal{M}$ satisfying (22). Then, for any half-invariant region $\mathcal{V}$ of $\mathcal{W}$ the triple $\mathcal{H}_{o}:=\left(\mathcal{H}_{o}, H_{o}(\mathcal{W}), H_{o}(\mathcal{V})\right)$ is a half-sided modular inclusion. The relative entropy function $S_{\phi}^{\mathcal{H}_{o}}(\lambda)=S^{\mathcal{R}_{o}\left(\mathcal{V}_{\lambda}\right)}\left(\varphi_{\phi} \| \varphi_{o}\right)$ is convex with respect to $\lambda$.

We point out that the extension condition (22) is verified in meaningful examples. In the Minkowski spacetime, it holds because of the Bisognano-Wichmann Theorem [5]. In the de Sitter spacetime, the de Sitter vacuum [19, 6] is $\beta_{G H^{-}}$KMS for every wedge $\mathcal{W}$ with respect to $\Lambda_{\mathcal{W}}$, where $\beta_{G H}$ is the Gibbons-Hawking inverse temperature.

A further relevant example is provided by the Kruskal spacetime; the analogy of the Bisognano-Wichmann setting of QFT for wedge regions in Minkowski spacetime and the for outer Schwarzschild region of Kruskal spacetime was noticed in [36]. The Hartle-Hawking-Israel state satisfies the Bisognano-Wichmann property, this result is essentially contained in [34], using the analysis in [22], as shown in the following.

Lemma 3.7. Let $\varphi_{o}$ be the Hartle-Hawking-Israel state of the scalar Klein-Gordon field on the Kruskal spacetime. Then

(i) the restriction of $\varphi_{o}$ to $\mathcal{A}\left(\mathcal{W}_{+}\right)$is $\beta_{H}-K M S$ with respect to $\Lambda$, with $\beta_{H}$ the Hawking inverse temperature;

(ii) $\varphi_{o}$ satisfies the Reeh Schlieder cyclicity property with respect to open, non-empty, relatively compact subregions;

(iii) $\varphi_{o}$ satisfies the Bisognano-Wichmann property

$$
\operatorname{ad}\left(J_{\mathcal{R}_{o}\left(\mathcal{W}_{+}\right)}\right) \mathcal{R}_{o}(\mathcal{O})=\mathcal{R}_{o}(\iota \mathcal{O}), \operatorname{ad}\left(\Delta_{\mathcal{R}_{o}\left(\mathcal{W}_{+}\right)}^{-i s}\right) \mathcal{R}_{o}(\mathcal{O})=\mathcal{R}_{o}\left(\Lambda_{2 \pi s} \mathcal{O}\right), \quad \mathcal{O} \subset \mathcal{W}_{+},
$$

where $\iota$ is the reflection symmetry of $\mathcal{M}$ on the $\mathbb{R}^{2}$ component: $\iota(t, x)=(-t,-x)$.

Proof. Sanders has shown in [34, Theorem 5.3] that the HHI state $\varphi_{o}$ is a pure quasifree Hadamard state on the Kruskal spacetime, invariant under the Killing flow $\Lambda$ and the reflection symmetry $\iota$, for the latter see comments after [34, Proposition 5.4]. More 
precisely, $\varphi_{o}$ is characterized by the fact that its restriction to the algebra of the right wedge $\mathcal{W}_{+}$is a KMS state $\varphi_{\beta_{H}}$ with respect to $\Lambda$, where $\beta_{H}$ is the Hawking inverse temperature. Furthermore, the restriction of $\varphi_{o}$ to the algebra of the double wedge $W_{-} \cup$ $W_{+}$coincides with the double-KMS state $\tilde{\varphi}_{\beta_{H}}$ that is associated with $\varphi_{\beta_{H}}$ accordingly to 22 .

Moreover, Kay showed that the double KMS-state $\tilde{\varphi}_{\beta_{H}}$ satisfies the BisognanoWichmann property on $W_{-} \cup W_{+}$[22]; actually this property is transferred to the HHI state $\varphi_{o}$ because, by [34, Proposition 5.4], the mapping

$$
W_{o}(f) \xi_{o} \mapsto \tilde{W}_{\beta_{H}}(f) \tilde{\xi}_{\beta_{H}}, \quad f \in C_{0}^{\infty}\left(\mathcal{W}_{-} \cup \mathcal{W}_{+}\right),
$$

gives a unitary operator between the Fock space of $\varphi_{o}$ and that of the double KMS state $\tilde{\varphi}_{\beta_{H}}$. By this unitary equivalence and the fact that $\tilde{\varphi}_{\beta_{H}}$ satisfies the Reeh-Schlieder property [34, Theorem 3.5], it follows that $\varphi_{o}$ satisfies the Reeh-Schlieder property too.

Acknowledgements. We are grateful to M. Sánchez for fruitful discussions and an explanation about the proof of Lemma 3.2.

We acknowledge the MIUR Excellence Department Project awarded to the Department of Mathematics, University of Rome Tor Vergata, CUP E83C18000100006.

\section{References}

[1] H. Araki, Relative entropy of states of von Neumann algebras, Publ. RIMS Kyoto Univ. 11, 809-833, (1976).

[2] H. ARAKI, L. Zsidó, Extension of the structure theorem of Borchers and its application to half-sided modular inclusions, Rev. Math. Phys. 17, 491-543, (2005).

[3] A.N. Bernal, M. Sánchez, On Smooth Cauchy Hypersurfaces and Geroch's Splitting Theorem, Comm. Math. Phys. 243, 46-470, (2003).

[4] A.N. Bernal, M. SÁnchez, Further Results on the Smoothability of Cauchy Hypersurfaces and Cauchy Time Functions, Lett. Math. Phys. 77, 183-197, (2006).

[5] J. Bisognano, E. Wichmann, On the duality condition for a Hermitean scalar field, J. Math. Phys. 16, 985-1007, (1975).

[6] H.J. Borchers, D. Buchholz, Global properties of vacuum states in de Sitter space, Annales del'I.H.P. Physique Theorique, 70, 23-40, (1999).

[7] H.J. Borchers, J. YNGvason, Modular groups of quantum fields in thermal states, J. Math. Phys. 40 no. 2, 601-624, (1999).

[8] R. Bousso, Z. Fisher, S. Leichenauer, A.C. Wall, Quantum focusing conjecture, Phys. Rev. D 93, 064044, (2016). 
[9] H. Bostelmann, D. Cadamuro, S. Del Vecchio, Relative entropy of coherent states on general CCR algebras, arXiv:2012.14401.

[10] R. Brunetti, D. Guido, R Longo, Modular localization and Wigner particles, Rev. Math. Phys. 14, 759-786, (2002).

[11] P. Camassa, R. Longo, Y. Tanimoto, M. Weiner, Thermal states in Conformal Field Theory. I, Comm. Math. Phys. 309 (2012), 703-735.

[12] P. Camassa, R. Longo, Y. Tanimoto, M. Weiner, Thermal states in Conformal Field Theory. II, Comm. Math. Phys. 315 (2012), 771-802.

[13] A.M. Candela, J.L. Flores, M. SÁnchez, M, Global hyperbolicity and PalaisSmale condition for action functionals in stationary spacetimes, Adv. Math. 218, 515-536, (2008).

[14] F. Ceyhan, T. Faulkner, Recovering the QNEC from the ANEC, Comm. Math. Phys. 377, 999-1045, (2020).

[15] F. Ciolli, R. Longo, G. Ruzzi, The information in a wave, Comm. Math. Phys. 379, 979-1000, (2020).

[16] C. Dappiaggi, G. Lechner, E. Morfa-Morales, Deformations of quantum field theories on spacetimes with Killing vector fields, Comm. Math. Phys. 305, 99-130, (2011).

[17] C. Dappiaggi, V. Moretti, N. Pinamonti, "Hadamard States from Light-Like Hypersurfaces", SpringerBriefs in Mathematical Physics, 25, Springer International Publishing, (2017).

[18] G.F. Ellis, S.W. Hawking, "The Large Scale Structure of Space-Time", Cambridge Monographs on Mathematical Physics, No.1, Cambridge University Press, (1973).

[19] G.W. Gibbons, S. Hawking, Cosmological event horizons, thermodynamics and particle creation, Phys. Rev. D15, 2738-2751, (1977).

[20] D. Guido, R. Longo, The conformal spin and statistics theorem, Comm. Math. Phys. 181, 11-35, (1996).

[21] S. Hollands, A. Ishibashi, News vs information, Class. Quant. Grav., 36, N. 19 (2019).

[22] B.S. KAY, The double-wedge algebra for quantum fields on Schwarzschild and Minkowski spacetimes. Comm. Math. Phys. 100, 57-81, (1985).

[23] B.S. KAY, A uniqueness result for quasifree KMS states, Helv. Phys. Acta 58, 1017-1029, (1985). 
[24] B.S. KAY, R.M. WALD, Theorems on the uniqueness and thermal properties of stationary, nonsingular, quasifree states on spacetimes with a bifurcate Killing horizon, Phys. Rep. 207, 49-136, (1991).

[25] F. Kurpicz, N. Pinamonti, R. Verch, Temperature and entropy-area relation of quantum matter near spherically symmetric outer trapping horizons, Lett. Math. Phys. 111 (2021), no. 4, Paper No. 110

[26] R. Longo, Notes for a quantum index theorem, Comm. Math. Phys. 222 (2001), $45-96$.

[27] R. Longo, Real Hilbert subspaces, modular theory, $S L(2, R)$ and CFT, in: "Von Neumann algebras in Sibiu", Theta Ser. Adv. Math., 10, 33-91, Theta, Bucharest, (2008).

[28] R. Longo, Entropy of coherent excitations, Lett. Math. Phys. 109 (2019), 258-260.

[29] R. Longo, Entropy distribution of localised states, Comm. Math. Phys. 373, 473$505,(2020)$.

[30] E. Minguzzi, Lorentzian causality theory, Living Rev. Relativ. 22, 3 (2019).

[31] V. Morinelli, Y. Tanimoto, B. Wegener, Modular operator for null plane algebras in free fields, arXiv:2107.00039

[32] C.W. Misner, K.S. Thorne, J.A. Wheeler, "Gravitation", W. H. Freeman and Co., San Francisco, California, (1973).

[33] F. Rocca, M. Sirugue, D. Testard, On a class of equilibrium states under the Kubo-Martin-Schwinger condition. II. Bosons, Comm. Math. Phys. 19, (1970) 119-141.

[34] K. Sanders, On the Construction of Hartle-Hawking-Israel States Across a Static Bifurcate Killing Horizon, Lett. Math. Phys. 105, 575-640, (2015).

[35] K. SAnders, Thermal equilibrium states of a linear scalar quantum field in stationary space-times, Int. J. Modern Phys. A 28, 1330010, (2013).

[36] G.L. SewelL, Quantum fields on manifolds: PCT and gravitationally induced thermal states, Annals Phys. 141 (1982) 201-224

[37] A. Strohmaier, The Reeh-Schlieder property for quantum fields on stationary spacetimes Comm. Math. Phys. 215, 105-118, (2000).

[38] R. Verch, Continuity of Symplectically Adjoint Maps and the Algebraic Structure of Hadamard Vacuum Representations for Quantum Fields on Curved Spacetime, Rev. Math. Phys. 9, 635-674 (1997).

[39] R.M. WALd, "General Relativity", University of Chicago Press, Chicago, IL, (1984). 